\title{
A PERSPECTIVE VIEW OF CREATING A SCHOOL OF THOUGHT FOR TRANSCENDENTAL PSYCHOLOGY IN ARMENIA
}

\begin{abstract}
The article shows a difference between the transcendental approach to the study of psychic phenomena from the methodologies dominating contemporary psychology. A possibility is shown in this context of establishing an indigenous school of transcendental psychology in Armenia.

Keywords: psyche, methodology, metaphysics, transcendental psychology, psychological school.

The development of science is intensified if it is actuated within a particular school of scientific thought. It is stipulated by the fact that the scientific trend determining the content of the school of thought will facilitate the association of adherents providing multiple augmentations of their creative potential, promoting a manifold aspect of the subject under study as well as introducing its own original image upon the International scene of Psychological Science.

The opportunity for creating a national or autonomous psychological school in Armenia came about in the late 80 s of the previous century but had not been realized. This opportunity, however, is feasible to date. What is meant is the psychological concept by A.I. Mirakian, presently designated as "Transcendental Psychology". We shall attempt here to reconstruct the course of history and to disclose the premises for the emergence of a new paradigm in psychology.

Initiation of Transcendental Psychology can be dated in 1987 when a doctoral thesis by A.I. Mirakian titled "The Constancy and Polyfunctionality of Perception" was sustained at the Psychological Institute of the Russian Educational Academy (Moscow) [3].

This work for the first time offers a methodology of a non-physical (transcendental) approach to the study of the generative process of perception and its underlying principles: What was then the need to transition to the new paradigm? Traditional psychology of perception has been leaning upon classical rationality [10] and has been aimed at resolving the question: "What is the way man perceives (reflects) the environment? This seemingly natural postulate will prompt the following premises of research:
\end{abstract}


- There exist the subject and the surrounding world. They interact.

- The world and the objects that it contains do not depend upon the subject.

- The objects possess multiple features within every modality of perception, which objects are similarly independent upon the subject perceiving them. The perception may introduce distortion; however, distortion may be done objectively to objects' properties independent of man.

- An object is simultaneously in possession of multiple properties, but their simultaneous scrutiny is impossible.

The quoted research premises for the process of perception looked quite natural and were postulated with no theoretical analysis or empirical argumentation.

The gradual emergence of classical rationality resulted, since the early 20th century to date, in recognizing the need to include the mediating links into the ratio of the "objectsubject" classical rationality in order to make the research complete, integral and objective. Those innovations have been implemented at diverse research levels of the perception process: the physical (instruments, research situation and environment), the physiological (physiological and chemical processes within the nervous system, neuronic networks and individual neurons as sensing units), the psychological (subjective features of personality). Meanwhile, this entire progress in perception research retains the extreme componential correlations of classical and non-classical rationality, i.e. "object" and "subject", hence retaining therewith all the four postulates quoted above. Thus, the perception psychology has developed in a way excluding the discovery of the universal principles of explication, that could integrate the endlessly variable patterns of research findings, whereby each one psychological theory of perception was ineffectively substituted by another. What has been presented so far, besides psychology of perception, is related to psychology as a whole? That is precisely the reason why V.M. Alahverdov saw the reason of this situation in that "all psychic concepts so far created contain some common defect. Therefore, it is expedient to find some common entity that is contained in all psychological concepts, and to try to reject it" [1, p. 11]

Along the way to rethink the problem of psychic reflection, A.I. Mirakian (1987) studied the arguments restricting the potential of its research, coming to the conclusion that those arguments are deeply rooted in the empirical reasoning of man, functioning within the realm of the products (phenomena) of psychic reflection. Those phenomena just determine the discrete character of the object and the subject, relevant to both classical and non-classical mentality. It is this mentality that enables determining only the type of relationships between the psychic phenomena without admitting penetration into the unconscious process of their 
generation to uncover the principles of those relationships.

This gives rise to a significant methodological problem of overcoming the constraint of the product-related approach that in turn leads to the need of both undertaking a philosophical analysis and forming an image of the world implementing the principles enabling the existence of psychic reality. This type of analysis, as we have pointed out in [15, c.11], has to start with a question "What should be the structure of the world that (in the course of evolution) could generate psyche? This question is based on several axioms:

1. The material world precedes the emergence of the psyche;

2. the world (in its entirety) and the principles of its development are not given to us directly (inference: therefore, their relevant presentations can be obtained through philosophical reasoning related to a specific section of philosophy, metaphysics, which includes the metaphysics of reality or ontology;

3. the principles of generating the new entities are everlasting and unchanged; what is relative is our empiric ideas about them (inference: therefore, they existed at the start of evolution and are active to date, implicitly and multifacetedly showing up and acquiring specific characteristics in structurally and procedurally organizing the physical, biological and physiological mechanisms of the organs of perception, as well as in the relevant products of psychic reflection).

The presented systems of postulates are quite differing, for they describe very different images of the world, differing realities, therefore, the theoretical structures based upon them (methodologies, theories, concepts, etc.) will indeed be related to different paradigms having dissimilar methodological recourses for interpreting the same psychological phenomena (what is reminiscent of the transition from the mechanics of classical physics to quantum mechanics).

Thus, A.I. Mirakian's suggestion is to study the principles of perception not through its phenomena given to us in experience, as accepted in modern psychology, but, having completed a speculative transition into the transcendental, supersensory domain of the perception process, using the a priori concepts, in order to try and determine the principles of the generative process of perception. That is why A.I. Mirakian will start his methodological research from the new and somewhat unusual image of the world. Characterizing this image of the world, A.I. Mirakian wrote: "with regard to the as yet non-completed act of reflection (not yet realized potential of reflection) our adopted infinite variety of material forms of matter is posing in a non-dismembered, integral form, as some homogeneity of globality. The notion of globality is introduced to indicate non-reflection and to point out a restricted potential of reflection with regard to what is reflected. The objects and their features as psychic shapes, 
according to the logic of reflection process as shape generation, have yet to come to be, to take a form, i.e. to be generated in the process of reflection (text emphasized by the author - R.N.), that can be interpreted as a process of regulating the globality, which process is aimed at retaining the shape and the functioning adequacy of a living system in the environment [4 c. 39-40]. The quoted citation yields several important inferences that are affecting our world image. Firstly, in reality, related to the transcendental domain of "the as yet unrealized potential of reflection", i.e. in reality existing prior to reflection, there exist no objects or their properties, but instead there exist material shapes constituting the nondismembered globality. Secondly, objects and their properties emerge as the products of the process of shape generation, i.e. already as psychic phenomena, thus demonstrating that the reality, perceived and recognized by man is nothing else but images generated by himself, experiences, views, etc. Thirdly, it is to be noted that what is on is not reflection, but rather shape generation, thus emphasizing the idea of generating something new, having no similarity (analogue, copy, conformity, or, by no means, identity with "the reflected", thus eliminating the problem of reflecting the reality.

It is becoming clear from what has been presented, that a completely new approach has been suggested to the methodology of psychic research, which approach cannot be inscribed into the framework of the existing methodologies. Without going into detail of theoretical constructs of the transcendental method, it can be noted that its fundamentals have been attested by extensive experimentation [16].

Working and residing in Moscow, A.I. Mirakian maintained permanent links with Armenia and the Armenian psychologists. In the late 80s, he arrived in Yerevan to organize his own school, but unfortunately, it could not be done. He returned to Moscow, where he was laid to rest in 1995.

Nonetheless, the concept of A.I. Mirakian was continued by the author of these lines and G.S. Esayan. Eventually there followed some publications on Transcendental Psychology [6], [7], [8], [11], [12], [13], [14], [15]; a Collection of articles by the Armenian and Russian psychologists has also been published in Yerevan: "The Psychology of Perception: a Transcendental View [17]; a doctoral thesis was sustained titled: "A Metaphysical Approach to the Methodology of Studying the Psychic Reflection of Reality" [9], substantiating for the first time that the fundamental principle of transcendental psychology is Metaphysics within the Aristotelian conception. Further on, G.S. Esayan sustained under our guidance a candidate thesis "The Contribution by A.I. Mirakian to the Development of the Concept of Psychic Reflection" [2]. It can thus be considered that a theoretical basis has been created in Armenia for developing this trend in Psychology. However, there is still a lot to be done. A 
textbook has yet to be written on Transcendental Psychology, and international conferences have to be convened on the problems of Philosophy of Psychology, work is to be done with the scientific personnel, applications to be extended of the ideas of Transcendental Psychology, etc., which will no doubt demand extensive organizing activities, financial investments, as well as the involvement of young enthusiastic psychologists (and not only psychologists), endowed with the philosophic and psychological make-up of mind. An essential moment of Transcendental Psychology is the establishment of International links with the concerned psychological communities.

\section{References}

Allahverdov V.M. (1993) An Experience in Theoretical Psychology. SPb., 198 p.

Esayan G.S. (2016) The Contribution by A.I. Mirakian to the Concept of Psychic Reflection. Thesis for Cand. of Psychol. Sci., Yerevan.

Mirakian A.I. (1987) The Constancy and Polyfunctionality of Perception. Thesis for Doc. of Psychol. Sci., Moscow.

Mirakian A.I. (1992) The Non-physical Principles of Psychic Reflection and Their Simulation. // The Principles of Process-generating Perception (a collective Study) edited by A.I. Mirakian. M.pp. 9-46.

Mirakian A.I. (1995) Elements of Transcendental Psychology of Perception // Philosophical Research, \#2. pp. 77 - 94.

Naghdyan R.M. (2007) Psychological Relativism of Reality. Yerevan, Sarvard, 90 p.

Naghdyan R.M. (2009) Psychological Relativism of Reality. Psychology of Philosophy. Yerevan, Nairi Publishers, 104 pp.

Naghdian R.M. (2013) Essays in Metaphysical Psychology. Yerevan, Nairi. 190 pp. (in Armenian).

Naghdyan R.M. (2015) A Metaphysical Approach to the Methodology of Research in the Psychic Reflection of Reality. Thesis for Doc. of Psychol. Sci. Yerevan.

Naghdyan R.M. (2016) Analysis of the Ratio of Classical and Non-classical Rationality in the Psychology of Perception (Psychophysics, Gestalt psychology) //Yerevan, Vestnik RAU, pp. 105-118.

Naghdyan R.M. (2017) The Riddle of the Psyche: Transcendental Psychology and Metaphysics. Yerevan, Lusabats Publ., $424 \mathrm{p}$.

Naghdyan R.M. (2018) Methodology of Transcendental Psychology. Yerevan, Lusabats Pub., $416 \mathrm{p}$. 
Nagdyan R.M. (2019) Transcendental Psychology (A Reading Book). Volume 1. Yerevan, Lusabats Pub., $424 \mathrm{p}$.

Naghdyan R.M. (2019) Transcendental Psychology (A Reading Book). Volume 2. Yerevan, Lusabats Pub., 424 p.

Naghdyan R.M. (2019) Transcendental Psychology (A Reading Book). Volume 3. Yerevan, Lusabats Pub., $424 \mathrm{p}$.

The Principles of Process-generating Perception (A Collective Study, Edited by A.I. Mirakian)( 1992) Moscow, $220 \mathrm{p}$.

Psychology of Perception: A Transcendental Perspective / Collection of Scientific Articles Edited by R. Naghdian and V.I. Panov (2017) Yerevan, Nairi Publ., 344 pp. 